\title{
Síntese e caracterização de nanopartículas de ouro coloidal com o látex como um agente redutor
}

\author{
Pedro Henrique da Conceição SILVA ${ }^{1 *}$, Anselmo Fortunato Ruiz RODRIGUEZ ${ }^{2}$ \\ ${ }^{1}$ Instituto Federal de Educação, Ciência e Tecnologia do Tocantins, Paraíso do Tocantis, TO, Brasil. \\ ${ }^{2}$ Universidade Federal do Acre, Rio Branco, AC, Brasil. \\ E-mail: pedro.silva@ifto.edu.br
}

\begin{abstract}
Recebido em abril/2018; Aceito em janeiro/2019.
RESUMO: Tradicionalmente as técnicas de síntese de nanopartículas utilizam solventes tóxicos, o que gera resíduos potencialmente nocivos tanto à saúde quanto ao meio ambiente. Buscando metodologias mais sustentáveis na síntese de nanopartículas várias pesquisas têm proposto a utilização de agentes biológicos ou parte deles, em substituição aos reagentes químicos tóxicos. Assim, este trabalho propôs sintetizar nanopartículas de ouro, utilizando síntese verde, ou seja, o látex da planta Sangue de Dragão (Croton lechleri Müll. Arg.) como agente redutor. A planta Sangue de Dragão é nativa dos países Peru e Bolívia, no Brasil ela é encontrada na região Norte, nos estados Rondônia, Acre e Amazonas. Para realizar a síntese de nanopartículas, em busca de condições de síntese e de aperfeiçoamento de parâmetros, utilizou-se a técnica de visão de nanopartículas em suspensão coloidal e estabilidade, com monodispersividade. Obtendo-se monodispersidade das nanopartículas de ouro, sendo formadas nanopartículas que se encontram no diâmetro entre $10 \mathrm{~nm}$ e $40 \mathrm{~nm}$. O potencial zeta esteve na faixa de da região de $+/-30 \mathrm{mV}$, também foi observado que as partículas se agregam com uma dispersão instável, no decorrer do tempo. A síntese verde utilizando o látex da planta Sangue de Dragão apresentou-se resultados compatíveis com as técnicas que utilizam os agentes tóxicos, porem sustentável e com baixo custo para a realização.
\end{abstract}

Palavras-chave: Croton lechleri, nanopartículas de ouro, ressonância plasmônica superficial.

\section{Synthesis and characterization of coloidal gold nanoparticles with latex as a reducing agent}

\begin{abstract}
Traditionally the techniques of synthesis of nanoparticles use toxic solvents, which generate residues potentially harmful to health and the environment. Looking for more sustainable methodologies in the synthesis of nanoparticles several researches has proposed the use of biological agents or part of them, replacing the toxic chemical reagents. Thus, this work proposed to synthesize gold nanoparticles, using green synthesis, that is, the latex of the Dragon Blood plant (Croton lechleri Müll. Arg.) As a reducing agent. The Dragon Blood plant is native to Peru and Bolivia, in Brazil it is found in the North region, in the states of Rondônia, Acre and Amazonas. To perform the synthesis of nanoparticles, searching for conditions of synthesis and improvement of parameters, the technique of nanoparticle vision in colloidal suspension and stability, with monodispersivity, was used. Obtaining monodispersity of the gold nanoparticles, being formed nanoparticles that are in the diameter between $10 \mathrm{~nm}$ and $40 \mathrm{~nm}$. The zeta potential was within the range of $+/-30 \mathrm{mV}$, it was also observed that the particles aggregate with an unstable dispersion over time. The green synthesis using the latex of the Dragon Blood plant presented results compatible with the techniques that use the toxic agents, but sustainable and with low cost to the realization.
\end{abstract}

Keywords: Croton lechleri, gold nanoparticles, superficial plasmon resonance.

\section{INTRODUÇÃO}

A Croton lechleri Müll. Arg., popularmente conhecida como Sangue de Dragão, é uma árvore heliófila, da família das Euphorbiaceae, monóica de caule cilíndrico irregular e coberto por uma casca lisa, de cor bege cinza, manchada de cores verde-azuladas. Essa espécie se distribui pelo oeste da Amazônia, muitas vezes sendo encontrada nas vertentes do Oceano Pacífico e também nas vertentes ocidentais de floresta úmida, nos países como Bolívia, Colômbia, Brasil, Equador e Peru, lugares com temperaturas variando entre $18{ }^{\circ} \mathrm{C}$ e $26^{\circ} \mathrm{C}$. A Sangue de Dragão possui um aroma agradável durante a noite, sendo usada como medicamentos no Peru, Bolívia e Brasil como cicatrizante e no tratamento de úlceras gástricas (LOPES et al., 2013). Alguns povos indígenas desses países usam a seiva (ou látex) dessa planta para estancar sangramentos, proteger lesões contra infecções, acelerar processos de cicatrizes, curar hemorroidas, úlcera estomacal, corrimento vaginal. O látex da Sangue de Dragão tem uma cor avermelhado que possui uma composição química bem rica em compostos fenólicos e alcalóides (taspina), diterpenos, flavon-3-óis (monômeros, dímeros e trímeros), proantocianidinas e também lignanas (LOPES et al., 2013).

A síntese de nanopartículas de ouro coloidal tem uma limitação, que é a estabilidade da dispersão diante a formação de estrutura do sólido. Quanto maior a estabilidade da dispersão, a agregação das partículas ocorre lentamente diminuindo o tamanho médio das mesmas. A redução de $\mathrm{Au}^{3+}$ para $\mathrm{Au}^{0}$ ocorre na presença de agentes redutores. A busca por agentes redutores naturais, além de contribuir com 0 desenvolvimento sustentável, pode promover maior estabilidade da dispersão no meio reacional, além de contribuir para o aumento de alguma atividade biológica já exibida pelo 


\section{Silva; Rodriguez}

composto natural das nanopartículas de ouro coloidal obtidas. Desta forma argumentos são importantes para o estudo da utilização do látex de Croton lechleri na redução do $\mathrm{Au}^{3+}$ para $\mathrm{Au}^{0}$, uma vezque no látex são encontrados compostos fenólicos que são doadores de elétrons, agindo assim como agente redutor.

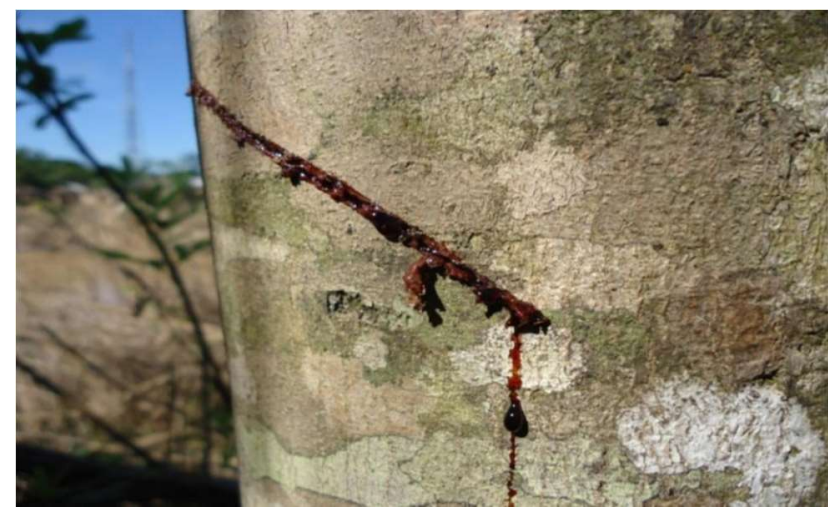

Figura 1. Caule de Croton lechleri nas proximidades do Rio Acre, no município de Xapuri/AC.

Figure 1. Croton lechleri snail near the Acre River, in the municipality of Xapuri/AC.

Há um intenso pico de absorção de radiação eletromagnética nos comprimentos de onda entre 500-600 nm devido à sua ressonância plasmônica de superfície (fenômeno que envolve a excitação coletiva de elétrons em um metal), que particularmente, as nanopartículas de ouro (AuNPs) apresentam. Esse pico ocorre quando a frequência do campo magnético se torna ressonante com o movimento desses elétrons. Devido a esse fato, observa-se uma variação de cor característica em uma dispersão de AuNPs, de amarelo avermelhado a marrom escuro, de acordo com o tamanho das nanopartículas produzidas e com a composição do meio em que elas estão dispersas.

Muitas técnicas fisicoquímicas tradicionalmente utilizadas na síntese de nanopartículas envolvem solventes tóxicos, limitando em diversas circunstâncias o amplo uso dessas nanopartículas no campo farmacêutico e clínico, e a gerando de resíduos potencialmente nocivos à saúde e ao meio ambiente (SALAM ET AL., 2012; CAUERHFF; CASTRO, 2013). Diante desse quadro, surge grande interesse na busca por rotas alternativas de síntese mais seguras e com menor impacto ao meio ambiente e, dentre elas, a síntese verde é uma abordagem promissora. Dada a relevância do tema exposto anteriormente, este trabalho tem como objetivo sintetizar nanopartículas de ouro, utilizando síntese verde, ou seja, o látex da planta Sangue de Dragão (Croton lechleri Müll. Arg.) como agente redutor.

\section{MATERIAL E MÉTODOS}

Seguindo as normas indicadas pela Universidade Federal do Acre - UFAC (AZEVEDO, 2008) no guia para extração do Sangue de Dragão, o látex extraído tem origem na cidade de Boca do Acre, no Estado do Amazonas, aproximadamente a 2,42 km subindo o rio Purus, a 3 metros da beira do rio Purus.

Foram preparadas diferentes soluções com concentrações diferentes do látex conforme segue na Tabela 1. Foi obtida uma solução coloidal pouca avermelhado devido à presença do látex da planta e, posteriormente, ao se colocar tetracloroáurico na proporção de $1 \mathrm{~mL}$ do látex em água destilada por 2,5 mL de ácido tetracloroáurico, formou-se uma suspensão de ouro coloidal.

Tabela. Relação entre soluto e solvente nas proporções analisadas Table 1. Relationship between solute and solvent in the proportions analyzed.

\begin{tabular}{cc}
\hline $\begin{array}{c}\text { Soluto (látex de Croton lechleri) } \\
\text { em mg }\end{array}$ & $\begin{array}{c}\text { Solvente (água destilada) } \\
\text { em mL }\end{array}$ \\
\hline 50 & 5 \\
75 & 5 \\
100 & 5 \\
150 & 5 \\
200 & 5 \\
\hline
\end{tabular}

Os íons $\mathrm{Au}^{3+}$ aparecem por meio do ácido tetracloroáurico, que é a forma ácida do cloreto de ouro. Nesse processo, observou-se mudança de coloração do vermelho para lilás, procedimento foi em torno de 5 minutos. A agregação das nanopartículas de ouro é instantaneamente evidenciada pela mudança de cor da solução e explicada pelo fato de onde a ressonância plasmônica de superfície (RPS) em ressonância com a luz incidente leva as oscilações de elétrons de mostra a diversidade de cores e tem sido devidamente explicada pela teoria de Maxwell-Garnet (MELO JUNIOR et al., 2012) segundo a Tabela 2.

Tabela 2. Indicação do tempo gasto para mudança completa da coloração no acréscimo do tetracloroáurico.

Table 2 - Indication of the time spent to completely change the coloration in the addition of tetrachlorouronic.

\begin{tabular}{cc}
\hline $\begin{array}{c}\text { Soluto (látex de Croton lechleri) } \\
\mathrm{em} \mathrm{mg} \text {, ou seja, } 10^{-3} \mathrm{~g}\end{array}$ & Tempo (s) \\
\hline 50 & 300 \\
75 & 240 \\
100 & 60 \\
150 & 15 \\
200 & 15 \\
\hline
\end{tabular}

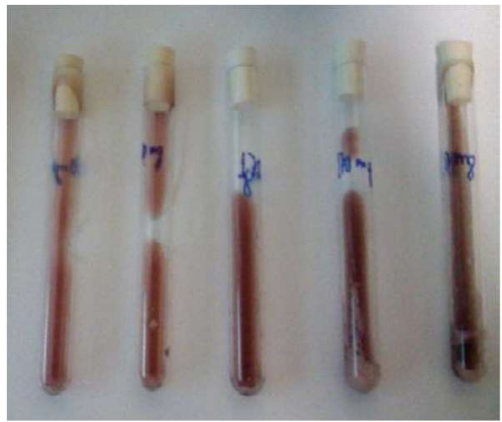

Figura 2. Látex misturada a água destilada.

Figure 2. Latex mixed with distilled water.

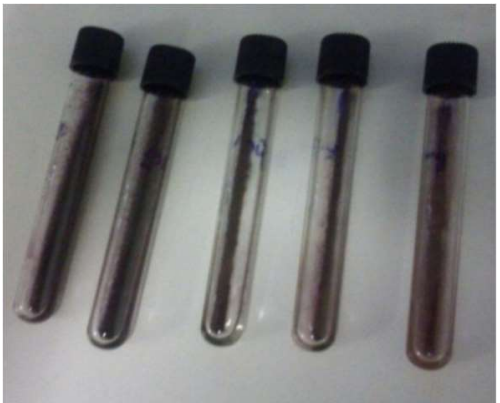

Figura 3. Látex misturada com o ácido tetracloroáurico. Figure 3. Latex blended with tetrachlorouric acid.

As medidas experimentais na espectroscopia Infravermelho (FTIR) com a amostra de látex centrifugada e 
uma alíquota do sobrenadante foi misturada com Brometo de Potássio $(\mathrm{KBr})$ formando pastilhas para serem medidas no equipamento que realiza espectrocopia de infravermelho chamado VERTEX 70/70v, a linha VERTEX 70 apresenta desempenho incomparável e versatilidade para aplicações de pesquisa e análises complexas, foram encontradas bandas em torno de $668 \mathrm{~cm}^{-1}$ a $3441 \mathrm{~cm}^{-1}$ sem acréscimo do tetracloroaúrico e após o acréscimo surgiram bandas de 1066 $\mathrm{cm}^{-1}$ a $3409 \mathrm{~cm}^{-1}$. Para Espectroscopia de UV-Vis foi utilizado o equipamento da Perkin Elmer LAMBDA UV/Vis/NIR Spectrophotometer 950 do Complexo BIONORTE do Laboratorio de Nanobiotecnologia da Universidade Federal do Acre - UFAC, onde foram encontradas as bandas de absorção na região de 335 à $350 \mathrm{~nm}$ sem acréscimo de tetracloroáurico e de 539 a $551 \mathrm{~nm}$ com o acréscimo. Para Microscopia Eletrônica de Transmissão (MET) foi utilizado o equipamento Microscópio Eletrônico de Transmissão (MET), Jeol, JEM2100, Thermoscientific, com o intuito de reproduzir as imagens das nanopartículas.

Usamos o programa Image J para coletar 130 partículas em tamanhos diferentes, ocorrendo maior número de frequências entre as proporções de tamanho entre $10-20 \mathrm{~nm}$ e entre 40 $50 \mathrm{~nm}$, que juntas aproximadamente $47 \%$ do total de nanopartículas de ouro.

\section{RESULTADOS}

Foram obtidos aproximadamente 1,0 L de látex de cor avermelhada apresentando aspecto límpido e pouco viscoso.

No espectro da espectroscopia Infravermelho, conforme Figura 4, é possível observar as bandas em $3441 \mathrm{~cm}^{-1}$ característica da deformação axial da hidroxila $\mathrm{OH}$, de alcoóis ou ácidos carboxílicos, no caso os grupos fenóis presentes no látex ou ácidos orgânicos. $\mathrm{O}$ alargamento da banda além de ser característica para o grupo $\mathrm{OH}$ é intensificado com a presença de água e, como o látex é uma solução aquosa. O pico em 2927 $\mathrm{cm}^{-1}$ é característica da deformação angular fora do plano da ligação C-H alifáticos. O pico em $1630 \mathrm{~cm}^{-1}$ é característica da deformação axial da ligação $\mathrm{C}=\mathrm{C}$ em aromáticos, assim como o pico em $668 \mathrm{~cm}^{-1}$ característica da deformação angular da ligação $\mathrm{C}-\mathrm{H}$ em aromáticos. As bandas observadas nas regiões de 1380 à $1450 \mathrm{~cm}^{-1}$ são características da deformação angular da ligação C-O em ácidos carboxílicos. As bandas em 1037 à $1200 \mathrm{~cm}^{-1}$ são características da deformação angular da ligação C-O em alcoóis e fenóis. (BARBOSA, 2007).

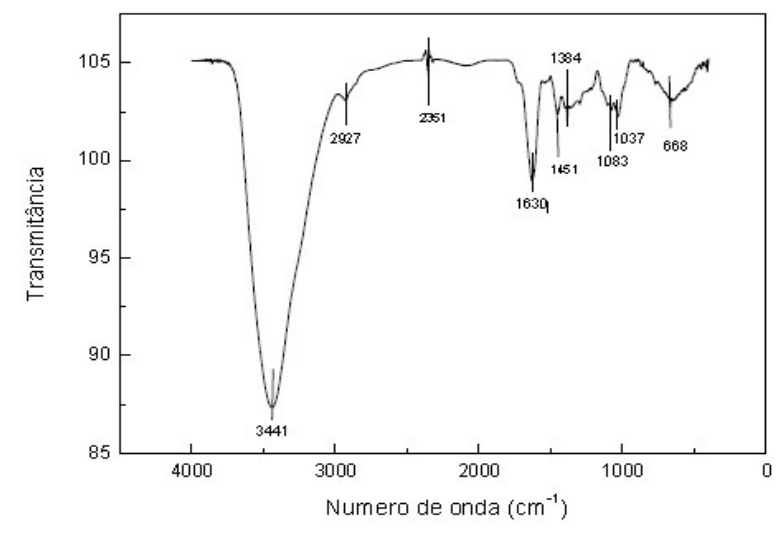

Figura 4. Espectro Infravermelho do látex sem tetracloroaúrico. Figure 4. Infrared Spectrum of Non-Tetrachlorouronic Latex.
Foi acrescentado o ácido tetracloroáurico e feita novamente a espectroscopia Infravermelho, conforme Figura 5 , e foi possível observar uma mudança nas bandas.

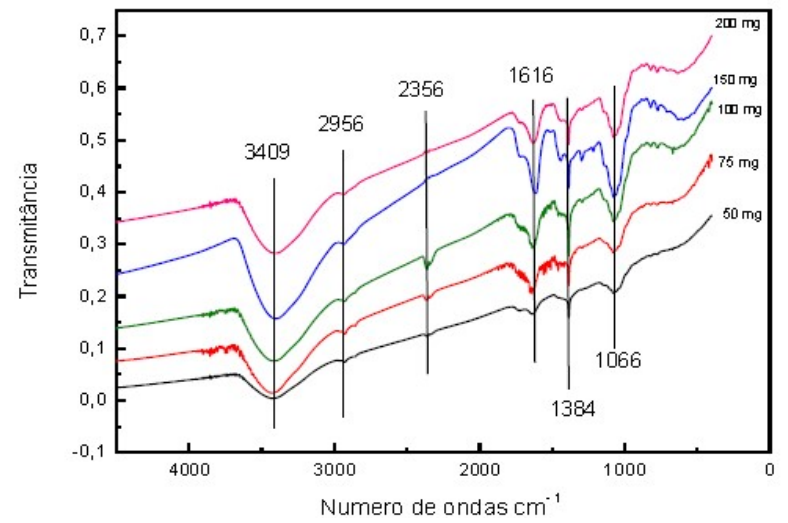

Figura 5. Espectros de infravermelho com tetracloroáurico nas medidas de $50 \mathrm{mg}, 75 \mathrm{mg}, 100 \mathrm{mg}, 150 \mathrm{mg}$ e $200 \mathrm{mg}$ de látex.

Figure 5. Infrared spectra with tetrachlorouronic in the measurements of $50 \mathrm{mg}, 75 \mathrm{mg}, 100 \mathrm{mg}, 150 \mathrm{mg}$ and $200 \mathrm{mg}$ of latex.

Foram realizadas espectroscopia de UV-Vis do látex em diferentes concentrações, conforme Figura 6, e observado que em todas as concentrações tivemos uma semelhança entre eles e que entre ocorreu bandas entre 450 a $500 \mathrm{~nm}$.

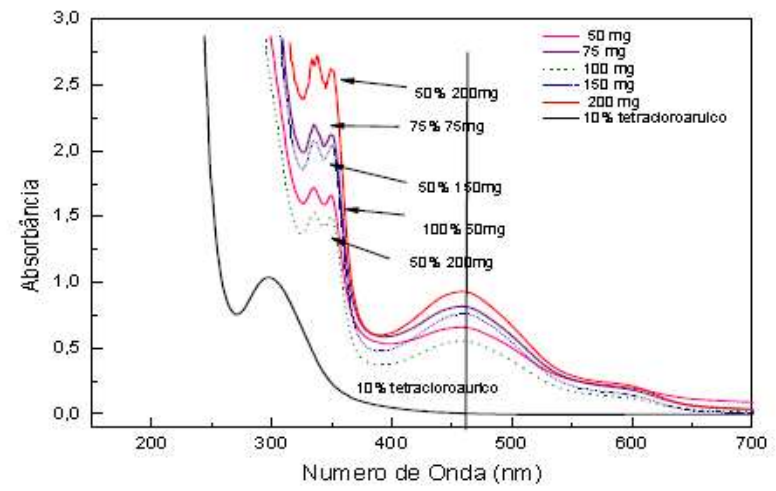

Figura 6. Espectroscopia de UV-Vis do látex a diferentes concentrações sem acréscimo do tetracloroaúrico.

Figure 6. UV-Vis spectroscopy of latex at different concentrations without addition of tetrachlorouronic.

Após o acréscimo do tetracloroaúrico, em Figura 7, observou-se em espectroscopia UV-Vis, houve um deslocamento da banda para 500 a $600 \mathrm{~nm}$.

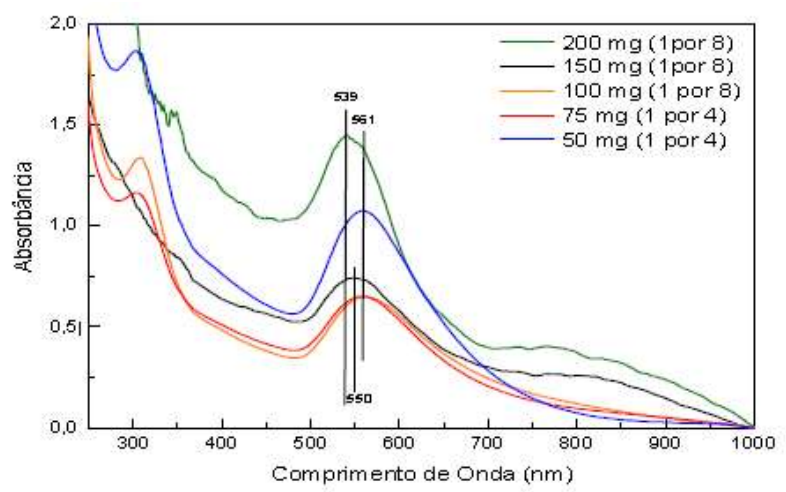

Figura 7. Espectro do UV-Vis com acréscimo do tetracloroáurico nas diferentes concentrações.

Figure 7. UV-Vis spectrum with addition of tetrachlorouronic at different concentrations. 


\section{Silva; Rodriguez}

Foram realizadas medidas de Polidispersão nas quatro amostras como mostra os resultados na Tabela 3, para Valores altos de índice de polidispersão indicam heterogeneidade do diâmetro das partículas em suspensão; variações nos valores de polidispersão em função do tempo indicam formação de populações de partículas com diâmetros que não existiam inicialmente, podendo ser decorrente de agregação ou quebra/degradação de partículas.

Além dos tamanhos das nano partículas, notou-se na Figura 8, o grande acúmulo de aglomerados de nanopartículas na medida de $50 \mathrm{mg}$, dificultando as medidas dos diâmetros das nanopartículas de ouro.

Tabela 3. Índice de Polidispersão nas 4 amostras.

Table 3. Polydispersion index in the 4 samples.

\begin{tabular}{|c|c|c|c|}
\hline \multicolumn{2}{|c|}{$\begin{array}{l}\text { Índice de Polidispersão de } 50 \mathrm{mg} \\
\text { Índice de Polidispersão (PdI) }\end{array}$} & \multicolumn{2}{|c|}{$\begin{array}{l}\text { Índice de Polidispersão de } 150 \mathrm{mg} \\
\text { Índice de Polidispersão (PdI) }\end{array}$} \\
\hline \multicolumn{2}{|c|}{0,494} & \multicolumn{2}{|c|}{0,919} \\
\hline \multicolumn{2}{|c|}{0,390} & \multicolumn{2}{|c|}{0,772} \\
\hline \multicolumn{2}{|c|}{0,304} & \multicolumn{2}{|c|}{0,513} \\
\hline Média: & 0,396 & Média: & 0,735 \\
\hline Desvio Padrão: & 0,095 & Desvio Padrão: & 0,200 \\
\hline \multicolumn{2}{|c|}{$\begin{array}{l}\text { Índice de Polidispersão de } 75 \mathrm{mg} \\
\text { Índice de Polidispersão (PdI) }\end{array}$} & \multicolumn{2}{|c|}{$\begin{array}{l}\text { Índice de Polidispersão de } 200 \mathrm{mg} \\
\text { Índice de Polidispersão (PdI) }\end{array}$} \\
\hline \multicolumn{2}{|c|}{0,747} & \multicolumn{2}{|c|}{1,000} \\
\hline \multicolumn{2}{|c|}{0,361} & \multicolumn{2}{|c|}{1,000} \\
\hline \multicolumn{2}{|c|}{0,417} & & \\
\hline Média: & 0,510 & Média: & 1,00 \\
\hline Desvio Padrão: & 0,170 & Desvio Padrão: & 0,00 \\
\hline
\end{tabular}

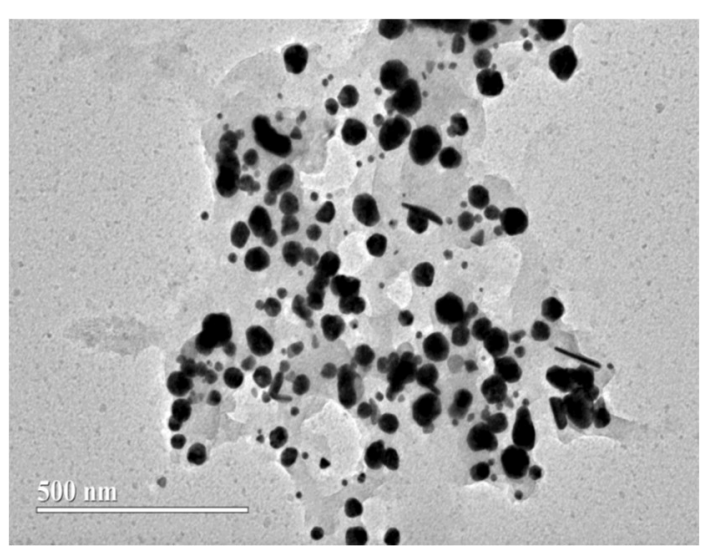

Figura 8. Imagem das nanopartículas de ouro com o Látex de Croton lechleri na medida de $50 \mathrm{mg}$.

Figure 8. Image of gold nanoparticles with Croton lechleri Latex at $50 \mathrm{mg}$.

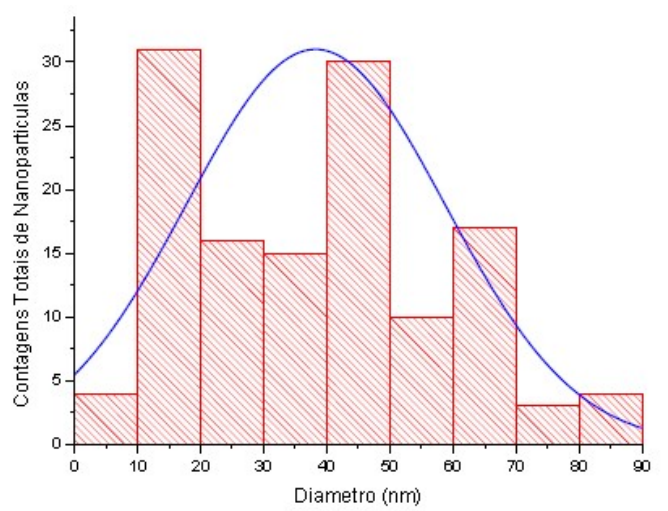

Figura 9. Histograma do diâmetro das nanopartículas de $50 \mathrm{mg}$. Figure 9. Histogram of the nanoparticle diameter of $50 \mathrm{mg}$.
Foram coletadas 172 nanopartículas de ouro em tamanhos bem diferenciados conforme Figura 10, o que comprova que as nanopartículas estão bem desagregadas e polidispersas. $\mathrm{O}$ resultado dessa pequisa mostra que quanto maior a concentração de látex, mais desagregadas e dispersas ficam as nanopartículas.

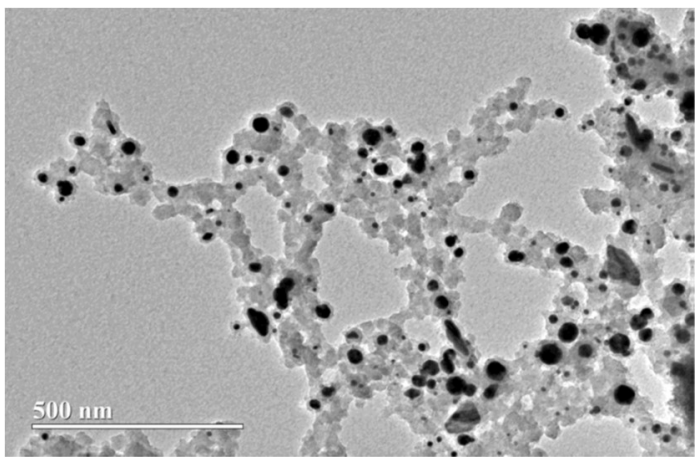

Figura 10. Imagem das nanopartículas de ouro com o Látex de Croton lechleri na medida de $200 \mathrm{mg}$.

Figure 10. Image of the gold nanoparticles with the Latex of Croton lechleri in the measurement of $200 \mathrm{mg}$.

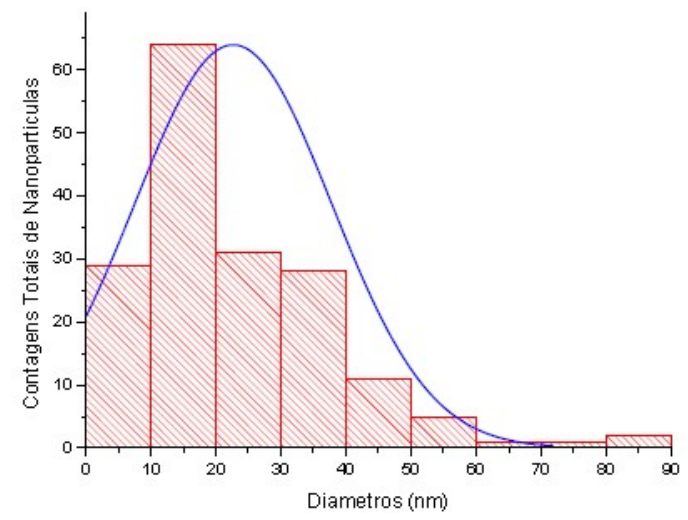

Figura 11. Histograma do diâmetro das nanopartículas de $200 \mathrm{mg}$. Figure 11. Histogram of the nanoparticle diameter of $200 \mathrm{mg}$.

\section{DISCUSSÃO}

A cor avermelhada do látex apresentada se dá em razão de ser composta por polifenóis substâncias caracterizadas por possuírem mais de um anel aromático, hidroxilados, as quais se mostram capazes de varrer radicais livres e aprisionar metais na formação de novos compostos, além disso esses grupos fenóis são cromóforos, ou seja, absorvem luz e, por serem sistemas altamente conjugados, a absorção se dá na região que vai do azul ao ultravioleta, apresentando a cor complementar observada que varia entre o amarelo e o vermelho (BATTESTIN et al., 2008).

As bandas que aparecem em $550 \mathrm{~nm}$ se referem às nanopartículas de $\mathrm{Au}^{0}$ sintetizadas e ocorre por que estas sofreram o efeito plasmônico, ou seja, ressonância para comprimentos de onda mais energéticos. Essa máxima da absorção é dependente do diâmetro médio das nanopartículas, possuindo intensidade maior quando as nanopatículas possuem o raio em torno de $20 \mathrm{~nm}$ (GEORGIEV et al., 2013).

As bandas entre 300 a $400 \mathrm{~nm}$ ainda permanecem. O deslocamento entre as bandas mais energéticas se dá devido aos diferentes tamanhos da nanopartículas de $\mathrm{Au}^{0}$, conforme já é esperado e relatado na literatura científica (VIOL, 2011).

Ocorre uma distribuição de cargas, criando um campo elétrico em torno da nanopartícula e até dentro dela mesma, ao ser excitada, o que afeta ao deslocamento da densidade 
eletrônica. Como resultado destes efeitos e da provocação das várias oscilações na nanopartícula, ocorre uma força de restauração ficando com vários elétrons em suas extremidades, esses elétrons interagem com a radiação que incide sobre a partícula criando oscilações harmônicas, o que resulta no surgimento da banda em $550 \mathrm{~nm}$ (NETTO-FERREIRA, 2015). $\mathrm{O}$ aumento no tamanho das partículas resulta em um deslocamento da banda plasmônica de superfície do metal para a região espectral de maior comprimento de onda.

Apresentaram menor estabilidade, evidenciada por menores valores de polidispersão e menores valores em módulo de potencial zeta (SOUZA, 2012). Os dados mostram que o tamanho das nanopartículas é variado (Multimodal) para todas as concentrações do látex que variam na formação de aglomerados de nanopartículas, ou seja, quanto mais látex menos aglomerados de nanopartículas . O que é notado é que o tempo é determinante na diminuição do índice de polidispersão (PD)I das amostras, onde a diminuição do tempo favoreceu a monodispersidade das nanopartículas de ouro preparadas sob agitação momentânea em agitador Vortex.

Observou-se na medida de Potencial Zeta que as partículas se agregam com uma dispersão instável, no decorrer do processo de análise. As nanopartículas de ouro, também possuem tamanhos variados, independente da concentração obedecendo à análise de potencial Zeta e de polidispersividade, ou seja, instabilidade incipiente se observam nas Imagens de Microscopia Eletrônica de Transmissão apresentando-se em forma agregadas e isoladas.

Além dos tamanhos das nano partículas, notou-se o grande acúmulo de aglomerados de nanopartículas, dificultando as medidas dos diâmetros das nanopartículas de ouro. A quantidade mais alta de látex proporciona um menor número de agregação das nanopartículas e também resulta em uma melhor homogeneidade na formação das nanopartículas.

\section{CONCLUSÕES}

$\mathrm{Na}$ Espectroscopia UV-vis observa-se a ocorrência da Ressonância Plasmônica, no momento em que ocorre a redução do ouro de $\mathrm{Au}^{3+}$ para $\mathrm{A}^{0}$, para ondas mais energéticas.

A oxidação é vista com a permanência das bandas entre 300 a $400 \mathrm{~nm}$, uma vez que todos os compostos presentes foram oxidados. O deslocamento entre as bandas mais energéticas se dá devido aos diferentes tamanhos das nanopartículas de $\mathrm{Au}^{0}$

No Potencial Zeta e o Índice de Polidispersão, apresentam nanopartículas estáveis, que tem a diminuição do tamanho dependente da concentração do látex, ou seja, quanto mais látex maior será o aumento da disponibilidade de sítios de reação. Obtivemos que as nanopartículas ficam mais tempo dispersas, mostrando estabilidade, o tamanho final das mesmas será menor, enquanto que a instabilidade da dispersão formará aglomerados rapidamente resultando em partículas maiores.

$\mathrm{O}$ resultado das imagens de MET nos mostra que o tamanho das nanopartículas é variado (Multimodal) para todas as medidas do látex que variam na formação de aglomerados de nanopartículas, ou sejam quanto mais látex menos aglomerados de nanopartículas. O que é notado é a duração do procedimento é determinante na diminuição do PDI das amostras, onde a diminuição do tempo favoreceu a monodispersidade das nanopartículas de ouro, sendo formadas mais partículas que se encontram no diâmetro entre $10 \mathrm{~nm}$ e $40 \mathrm{~nm}$. Comprovando assim, a formação de nanopartículas de ouro coloidal usando como agente redutor o látex de Croton lechleri.

\section{REFERENCIAS}

AZEVEDO, K.; ALECHANDRE, A.; LIMA, Á.; LEITE, A.; MELO, T.; COSTA, J.; PEREIRA, M. A.; CAMPOS, C. A.; LIMA, A. de. Guia para a extração de sangue de grado (Croton lechleri Müll. Arg.): recomendações técnicas para a extração de látex de sangue de grado (sangue de dragão). Rio Branco: Usaid-Ipam, 2008. 28p.

BATTESTIN, V.; MATSUDA, L. K.; MACEDO, G. A. Fontes e aplicações de taninos e tanases em alimentos. Alimentos e Nutrição, v. 15 n. 1, p. 63-72, 2008.

CAUERHFF, A.; CASTRO, G. R. Bionanoparticles, a green nanochemistry approach. Electronic Journal of Biotechnology, v. 16, n. 3, 2013. DOI: https://dx.doi.org/10.2225/vol16-issue3-fulltext-3

BARBOSA, L. C. de A. Espectroscopia no infravermelho: na caracterização de compostos orgânicos. Viçosa: Editora UFV, 2007. 189 p.

GEORGIEV, P.; BOJINOVA; A.; KOSTOVA, B.; MOM. Implementing atomic force microscopy (AFM) for studying kinetics of gold nanoparticle's growth. Colloids and Surfaces A: Physicochemical and Engineering Aspects, Amsterdam, v. 434, p. 154-163, 2013. DOI: https://dx.doi.org/10.1016/j.colsurfa.2013.05.064

LOPES, T. V.; FÉLIX, S. R.; DE VARGAS SCHONS, S.; OLIVEIRA NOBRE, M. (2013). Dragon's blood (Croton lechleri Mull., Arg.): an update on the chemical composition and medical applications of this natural plant extract. Revista Brasileira de Higiene e Sanidade Animal, Fortaleza, v. 7, n. 2, p. 167-191. DOI: http://dx.doi.org/10.5935/1981-2965.20130016

MELO JUNIOR, M. A.; SANTOS, L. S. S.; GONCALVES, M. do C.; NOGUEIRA, A. F. Preparação de nanopartículas de prata e ouro: um método simples para a introdução da nanociência em laboratório de ensino. Química Nova, São Paulo, 2012. DOI: http://dx.doi.org/10.1590/S0100-40422012000900030

NETTO-FERREIRA, J. C. Reações Catalíticas Empregando o Efeito Plasmônico de Nanopartículas Metálicas Suportadas. Revista Virtual de Química, v.7, n.1, p.165217, 2015. http://dx.doi.org/10.5935/1984-6835.20150010

SALAM, H. A; WANG, D.; LI, Y. Green route for nanoparticle synthesis. Chemical Society Reviews, London, v. 1, n. 5, p. 6, 2012. DOI: https://dx.doi.org/10.1039/c4cs00363b

SOUZA, P. M. S.; LOBO, F A.; ROSA, A. H.; FRACETO, L. F. Desenvolvimento de nanocápsulas de poli-ecaprolactona contendo o herbicida atrazina, Química Nova, São Paulo, v. 35, n. 1, p. 132-137, 2012. DOI: http://dx.doi.org/10.1590/S0100-40422012000100024

VIOL, L. C. de S.; SILVA, F. O.; FERREIRA, D. L.; ALVES, J. L. A.; SCHIAVON, M. A. Size-selective precipitation in colloidal semiconductor nanocrystals of CdTe and CdSe: a study by UV-VIS spectroscopy. Química Nova, v. 34, n. 4, p. 595-600, 2011. DOI: http://dx.doi.org/10.1590/S0100-40422011000400008 